\title{
TT-1, an analog of melittin, triggers apoptosis in human thyroid cancer TT cells via regulating caspase, Bcl-2 and Bax
}

\author{
LANLAN WAN ${ }^{1,2}$, DAQI ZHANG ${ }^{3}$, JINNAN ZHANG $^{4}$ and LIQUN REN ${ }^{2}$ \\ ${ }^{1}$ Department of Anesthesiology, The Second Hospital of Jilin University, Changchun, Jilin 130033; ${ }^{2}$ Department of \\ Pharmacology and Toxicology, Jilin University School of Pharmaceutical Sciences, Changchun, Jilin 130021; \\ ${ }^{3}$ Department of Thyroid Surgery, China-Japan Union Hospital of Jilin University, Jilin Provincial Key Laboratory of \\ Surgical Translational Medicine; ${ }^{4}$ Department of Neurosurgery, China-Japan Union Hospital of \\ Jilin University, Changchun, Jilin 130033, P.R. China
}

Received January 2, 2016; Accepted June 22, 2017

DOI: $10.3892 / \mathrm{ol} .2017 .7366$

\begin{abstract}
Melittin is a 26 amino acid residue antimicrobial peptide with known antitumor activity. In the present study, a novel peptide TT-1, derived from melittin and contained only 11 amino acids, was designed, and its antitumor effect was investigated. The present study is aimed to elucidate the effects and relative mechanisms of TT-1 on a human thyroid cancer cell line (TT) in vitro and in vivo. Cell viability assays, Annexin V/propidium iodide assays, western blotting and quantitative reverse transcription polymerase chain reaction were performed. Furthermore, a tumor-xenograft model was established to investigate the apoptotic mechanisms of TT-1 on TT cells. The results obtained indicated that TT-1 was able to suppress the proliferation of TT cells and exhibited low cytotoxicity to normal thyroid cells in vitro. The apoptotic rates of TT cells were also increased following TT-1 treatment. Additionally, TT-1 stimulated caspase-3, caspase- 9 and Bax, and inhibited B-cell lymphoma 2 mRNA and protein expression. Finally, it was also demonstrated that TT-1 is able to markedly suppress tumor growth in a TT-bearing nude mouse model. In summary, TT-1 may inhibit the proliferation of TT cells by inducing apoptosis in vitro and in vivo, indicating that TT-1 may be a potential candidate for the treatment of thyroid cancer.
\end{abstract}

Correspondence to: Professor Liqun Ren, Department of Pharmacology and Toxicology, Jilin University School of Pharmaceutical Sciences, 1266 Fujin Road, Changchun, Jilin 130021, P.R. China

E-mail: renlq_ren@sina.com

Dr Daqi Zhang, Department of Thyroid Surgery, China-Japan Union Hospital of Jilin University, Jilin Provincial Key Laboratory of Surgical Translational Medicine, 126 Xiantai Street, Changchun, Jilin 130033, P.R. China

E-mail: daqizhang@yeah.net

Key words: TT-1, TT cells, apoptosis, caspase, Bax, B-cell lymphoma 2

\section{Introduction}

Human cancer remains a leading cause of mortality worldwide despite recent advances in therapeutic methods (1). Incidences of thyroid cancer have steadily increased, and the disease has become the most prevalent endocrine malignancy worldwide (2). Thyroid cancer has an age-standardized incidence distribution and is estimated to occur in 9.1 per 100,000 females and 2.9 per 100,000 males in developed countries (3) According to the most recent World Health Organization classification of thyroid tumors, the disease classification includes papillary thyroid cancer (PTC), follicular thyroid cancer (FTC), poorly differentiated thyroid cancer, and anaplastic thyroid cancer (4). PTC and FTC are differentiated thyroid cancers and account for $>90 \%$ of all thyroid malignancies (5). The classic treatment of thyroid cancer is a total thyroidectomy followed by radioiodine treatment (5). Chemotherapy and biochemotherapy have also been used in the treatment of advanced melanoma. However, these treatments lack sufficient efficacy, and their toxicity and significant side effects greatly restrict their clinical application (6). Therefore, it is necessary to find an efficient and low-toxic anticancer drug for patients with thyroid cancer.

Antimicrobial peptides (AMPs) are natural peptides found in a wide range of organisms, including prokaryotes, insects, fish, amphibians and mammals (7). AMPs are cationic and amphiphilic molecules and have a wide range of characteristics based on peptide sequences, sizes, structural motifs and the presence of disulfide bonds (8). The molecules possess broad antimicrobial activity by binding to target bacteria to disrupt the membrane structure or by inhibiting fundamental bacterial metabolism (9). In addition to the antimicrobial activity, previous studies have also demonstrated that AMPs can selectively kill cancer cells because the membrane proteins of cancer cells are negatively charged due to glycosylation (10).

Melittin, a major peptide component of bee venom (Apis mellifera), is a cationic peptide comprising a small linear peptide of 26 amino acid residues $(11,12)$. Melittin was demonstrated to exert broad antimicrobial activity against bacteria by intercalating into cell membranes and causing changes in the membrane properties (13). Previous studies demonstrated that melittin also has in vitro anticancer activities against several 
cancerous cells, including renal, lung, breast and bladder cells $(14,15)$. However, high synthesis cost, high cytotoxicity and low stability have prevented the development of melittin as a promising anticancer agent $(16,17)$.

TT-1 is a mutant of melittin generated by a reduction of the peptide chain length and replacing glycine residues with lysine residues. The peptide sequence was changed from 'GIGAVLKVLTTGLPALISWIKRKRQQ' to 'KIKAVLKVL TT', which contained only 11 amino acids. The TT-1 mutant retained the amino-terminal active site region of melittin, has an increased hydrophobicity and a decreased net charge, which indicates a higher stability and lower toxicity compared with melittin $(18,19)$. The present study investigated the activity and the mechanism of TT-1 in the treatment of human thyroid cancer TT cells. The results revealed that TT-1 suppressed the proliferation of TT cells by inducing apoptosis via upregulation of Bax, downregulation of B-cell lymphoma-2 (Bcl-2) and the activation of caspase- 3 and -9 at transcriptional and translational levels. These interferences further inhibited TT tumor growth in nude mice. These results highlighted the therapeutic potential of TT-1 in thyroid cancer.

\section{Materials and methods}

Peptide synthesis. TT-1 (KIKAVLKVLTT) was synthesized by GL Biochem (Shanghai, China) via a stepwise solid phase methodology. The resulting peptide was purified via a Sephadex gel column and high-performance liquid chromatography to achieve a $>98 \%$ homogeneity of the purified peptide. The peptide information was analyzed using the antimicrobial peptide database (http://aps.unmc.edu/AP/main.html). After entering the home page of the website, 'Calculation \& Prediction' was chosen and the amino acid sequence of the peptide was entered in the newly opened web page, which then displays information on the peptide.

Cell lines and regents. The human thyroid cancer cell line TT and a normal human thyroid follicular epithelial cell line Nthy-ori3-1, obtained from the American Type Culture Collection (Manassas, VA, USA), were maintained as previously described (20). Briefly, TT and Nthy-ori3-1 were cultured in RPMI 1640 medium (Gibco; Thermo Fisher Scientific, Inc., Waltham, MA, USA) supplemented with $10 \%$ fetal bovine serum provided by Gibco (Thermo Fisher Scientific, Inc.), $100 \mathrm{U} / \mathrm{ml}$ penicillin, and $100 \mathrm{U} / \mathrm{ml}$ streptomycin. The cells were cultured at $37^{\circ} \mathrm{C}$ with $5 \% \mathrm{CO}_{2}$. MTT, sodium pyruvate and dimethyl sulfoxide (DMSO) were obtained from Sigma-Aldrich (Merck KGaA, Darmstadt, Germany). Annexin V-fluorescein (AV) and propidium iodide (PI) were purchased from Invitrogen (Thermo Fisher Scientific, Inc.). Fluorometric assay kits for measuring the activities of caspase- 3 and 9 were purchased from Cell Signaling Technology, Inc. (Danvers, MA, USA). Monoclonal antibodies against Bax (cat. no. sc-52895; dilution: 1:1,000) and Bcl-2 (cat. no. sc-509; dilution: 1:1,000) were purchased from Santa Cruz Biotechnology, Inc. (Dallas, TX, USA). The easy Plus Mini kit, iScript Select cDNA Synthesis kit, SyberGreen qPCR primer and iCycleriQ ${ }^{\mathrm{TM}}$ multicolor real time PCR detection system were purchased from Nanjing KeyGen Biotech Co., Ltd. (Nanjing, China).
Cell viability assay. To evaluate the effects of TT-1 on TT cells and Nthy-ori3-1 cells, the MTT assay was conducted as previously described (21). Briefly, the cells were cultured in 96-well plates at a density of $5 \times 10^{3}$ cells/well and allowed to attach for $12 \mathrm{~h}$. Different concentrations (0-32 $\mu \mathrm{g} / \mathrm{ml})$ of TT-1 were added to the cells, and the cells were further incubated for 24,48 and $72 \mathrm{~h}$ at $37^{\circ} \mathrm{C}$. Following incubation, the cells were incubated with MTT solution $(5 \mu \mathrm{g} / \mathrm{ml})$ for $4 \mathrm{~h}$ at $37^{\circ} \mathrm{C}$ followed by the addition of $150 \mu \mathrm{l}$ DMSO per well and then shaken for $5 \mathrm{~min}$ prior to measuring the absorbance at $490 \mathrm{~nm}$.

Cell apoptosis assay. Cell apoptosis assays (22) were conducted by double staining with $\mathrm{AV}$ and propidium iodide (PI) kit (ebioscience; Thermo Fisher Scientific, Inc.) to investigate whether TT-1 is able to induce apoptosis in TT cells. TT cells $\left(5 \times 10^{5}\right.$ cells/well $)$ were placed in 6 -well plastic plates $24 \mathrm{~h}$ prior to the TT-1 treatments. Following the replacement of medium supernatant, various concentrations of TT-1 $(0-8 \mu \mathrm{g} / \mathrm{ml})$ diluted in PBS were added. Following incubation at $37^{\circ} \mathrm{C}$ with $5 \% \mathrm{CO}_{2}$ for $48 \mathrm{~h}$, the cells were harvested. The $\mathrm{AV} / \mathrm{PI}$ assays were performed following the manufacturer's instructions. Subsequently, the cell samples were analyzed by flow cytometry (BD Biosciences, San Jose, CA, USA) and the data was analyzed by the flowjo 9 software (FlowJo LLC, Ashalnd, OR, USA).

Western blotanalysisfor Bax and Bcl-2 protein expression. The effects of TT-1 on Bax and Bcl-2 expression of TT cells were examined by western blotting as previously described (23). TT cells treated with or without TT-1 $(0-8 \mu \mathrm{g} / \mathrm{ml})$ for $48 \mathrm{~h}$ were homogenized in lysis buffer and centrifuged at $10,000 \mathrm{x} \mathrm{g}$ for $20 \mathrm{~min}$ at $4^{\circ} \mathrm{C}$. Then, the supernatants were analyzed for protein content by BCA assay. Equal amounts of protein sample $(50 \mu \mathrm{g})$ were loaded and separated by $12 \%$ SDS-PAGE and electrically transferred onto polyvinylidene fluoride membranes (EMD Millipore, Billerica, MA, USA). Afterward, the membranes were blocked in TBST supplemented with $5 \%$ bovine serum albumin for $2 \mathrm{~h}$ at $37^{\circ} \mathrm{C}$ followed by incubation at $4^{\circ} \mathrm{C}$ overnight in primary anti-Bax and anti-Bcl-2 antibodies, and finally incubation at $37^{\circ} \mathrm{C}$ for $1 \mathrm{~h}$ with secondary antibodies [goat anti-mouse $\operatorname{IgG}(\mathrm{H} \& \mathrm{~L})$ (HRP), cat. no. KC-MM-035; goat anti-rabbit IgG (H\&L) (HRP) or cat. no. KC-RB-035; both diluted to 1:5,000; both Zhejiang Kangchen Biotech Co., Ltd., Wuhan, China]. Following washing with TBST three times for $10 \mathrm{~min}$, the membranes were exposed by a chemiluminescence (ECL) detection kit. Bio-Imaging Image Lab 6.0 System software (Image Lab, Hercules, CA, USA) was used to detect the blot. Meanwhile, all blots were stripped and reprobed using a monoclonal anti- $\beta$-actin antibody (Santa Cruz Biotechnology, Inc.; grant no. sc-47778; dilution 1:1,000) to determine whether the proteins were equally loaded.

Determining the activity of caspase-3 and -9 . The effects of TT- 1 on the activity of caspase- 3 and -9 in TT cells were determined using a fluorometric assay kit (Calbiochem; EMD Millipore) according to the manufacturer's protocol as previously described (24). Briefly, TT cells were treated with TT-1 $(0-8 \mu \mathrm{g} / \mathrm{ml})$ for $48 \mathrm{~h}$ and harvested prior to the preparation of cell lysates. Then, the reaction buffer and the corresponding fluorogenic peptide substrate, Ac-DEVD-AMC (caspase-3) 
A

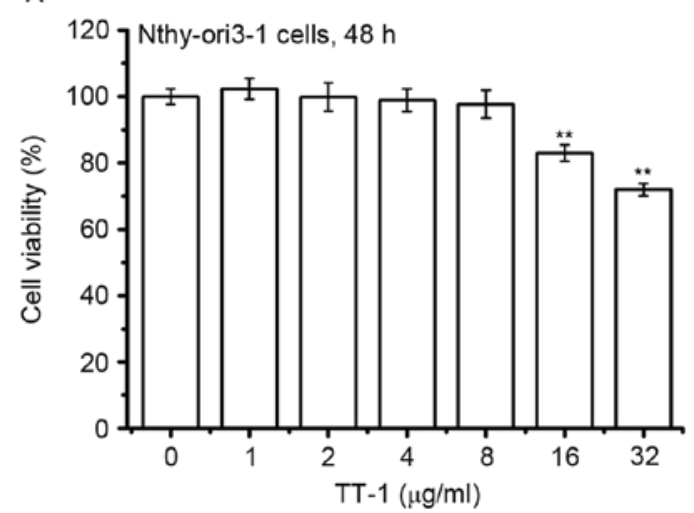

B

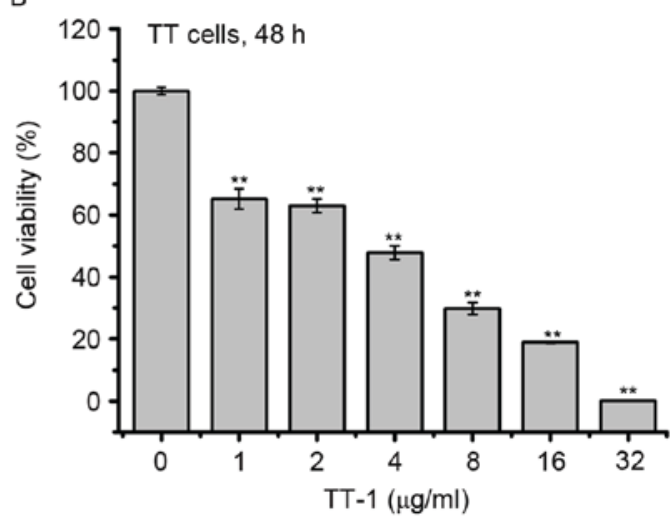

C

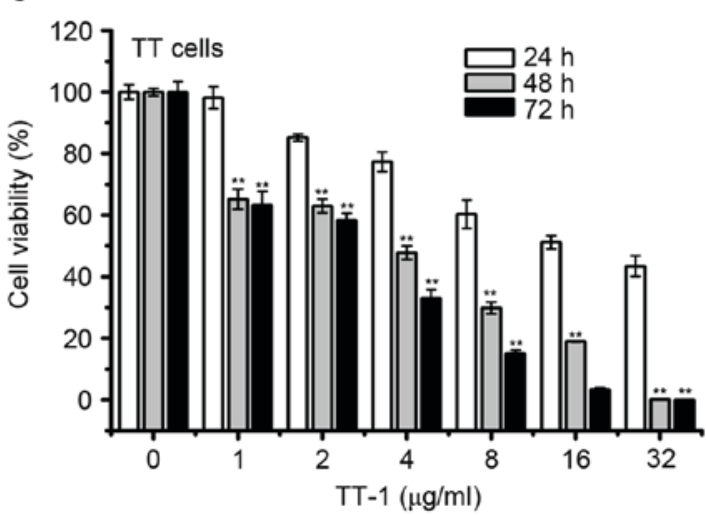

Figure 1. TT-1 selectively suppresses the proliferation of TT cells in vitro. TT or Nthy-ori3-1 cells were treated with different concentrations of TT-1 for 24 , 48 and $72 \mathrm{~h}$. Cell viability was measured using MTT assay. (A) TT-1 exhibited lower cytotoxic effects on Nthy-ori3-1 cells compared with TT cells. (B) TT-1 treatment inhibited cell viability (over $48 \mathrm{~h}$ ) in a dose-dependent manner. (C) The inhibition of proliferation of TT cells by TT-1 in a dose and time-dependent manner. Values are presented as the mean \pm standard deviation for three independent experiments with six replicates for each experiment. ${ }^{* *} \mathrm{P}<0.01, \mathrm{compared}$ with the untreated control at each time point.

and Ac-LEHD-AMC (caspase-9), were added to the cell lysates and incubated for $2 \mathrm{~h}$ at $37^{\circ} \mathrm{C}$ in the dark. The activity of caspase-3 and -9 in TT-1-treated TT cells were investigated using a microplate reader at $390 \mathrm{~nm}$ (excitation) and $500 \mathrm{~nm}$ (emission).

Quantitative reverse transcription polymerase chain reaction (RT-qPCR). The effects of TT-1 on the Bax, Bcl-2, caspase-3 and caspase-9 RNA expression in TT cells were examined by RT-qPCR as previously described $(25,26)$. Total RNA was extracted from TT cells with or without TT-1 treatment $(0-8 \mu \mathrm{g} / \mathrm{ml}$ for $48 \mathrm{~h}$ ) by using Trizol reagent and then purified with an RNeasy Mini kit (Qiagen GmbH, Hilden, Germany). Afterward, qPCR was conducted with an ABI PRISM 7300 sequence detection system (Applied Biosystems; Thermo Fisher Scientific Inc.), and 3 wells were used for each reaction. The relative mRNA expression was calculated using the comparative $\mathrm{Cq}\left(2^{-\Delta \Delta \mathrm{Cq}}\right)$ method $(27)$. The primers were as follows: Caspase-3 forward, 5'-AGGAAGGTGGCAACG-3' and reverse, 5'-CGCCAAATCTTGCTAAT-3'; caspase-9 forward, 5'-GGCTGTCTACGGCACAGATGGA-3' and reverse, 5'-CTGGCTCGGGGTTACTGCCAG-3'; Bax forward, 5'-GGCCCACCAGCTCTGAGCAGA-3' and reverse, 5'-GCCACGTGGGCGGTCCCAAAGT-3'; Bcl-2 forward, 5'-GTGGAGGAGCTCTTCAGGGA-3' and reverse, 5'-AGG CACCCAGGGTGAGCAA-3'.
TT-xenograft mouse model and TT-1 administration in vivo. Ethical approval for the present study was obtained from the Institutional Animal Care and Use Committee at Jilin University (Jilin, China). A total of 40 4-week-old nude mice (male; weight, 16-18 g) were purchased from the Jilin University Bethune School of Medicine and housed in a rodent facility at $22^{\circ} \mathrm{C}$ with a $12 \mathrm{~h}$ light-dark cycle. The mice were provided with continuous standard rodent chow and water. TT cells $\left(1 \times 10^{7}\right)$ were collected in the logarithmic phase of growth, diluted with normal saline and then inoculated intradermally into the hind flank. The tumors were inoculated for 12 days. The nude mice were randomly ( $n=10$ per group) divided into four groups, a model control group administered with normal saline and three TT-1-treated groups, which were administered at $0.04,0.2$ or $1 \mathrm{mg} / \mathrm{kg}$ body weight with intra-tumor injection three times a week. At the indicated time points, the mice from all the groups were sacrificed by overdose of anesthetics $24 \mathrm{~h}$ following final administration. Tumor weights of the mice were measured. Additionally, the tumor volume of each mouse was measured every three days during the treatment. The antitumor activities were expressed as inhibitory rate (\%) and calculated as $[(\mathrm{A}-\mathrm{B}) / \mathrm{A}] \times 100 \%$, where $\mathrm{A}$ and $\mathrm{B}$ were the mean tumor weight of the model and TT-1 groups, respectively. The tumor volumes (TV) were calculated using the following formula: $T V=1 / 2 \mathrm{xaxb}^{2}$, where $\mathrm{a}$ and $\mathrm{b}$ are the long and short diameters of the tumors in each mouse, respectively. 
Statistical analysis. All experiments conducted in the present study were performed in triplicate. The data are presented as the mean \pm standard deviation. Statistical analysis was performed by Student's t-test. $\mathrm{P}<0.05$ was considered to indicate a statistically significant difference.

\section{Results}

TT-1 selectively inhibits the viability of TT cancer cells but not normal human thyroid cells. To determine the cytotoxic activity of TT-1, MTT assay was conducted. As shown in Fig. 1A, the viabilities of normal human thyroid follicular epithelial cells Nthy-ori3-1 did not decrease in response to TT-1 treatment at concentrations up to $8 \mu \mathrm{g} / \mathrm{ml}$ for $48 \mathrm{~h}$. Furthermore, treatment with TT-1 significantly inhibited the proliferation of TT cells in a dose and time-dependent manner (Fig. 1B and C). The $50 \%$ inhibitory concentrations $\left(\mathrm{IC}_{50}\right)$ of TT- 1 on TT cells were $18.23 \pm 2.81,3.87 \pm 0.34,2.76 \pm 0.32 \mu \mathrm{g} / \mathrm{ml}$ at 24,48 and $72 \mathrm{~h}$, respectively. Specifically, at $4 \mu \mathrm{g} / \mathrm{ml} \mathrm{TT}-1$, the viability of TT cells at $48 \mathrm{~h}$ decreased to $47.8 \%$, which was much lower compared with the viability of Nthy-ori3-1 cells. The results showed that TT-1 exhibited high cytotoxicity on TT cancer cells and low cytotoxicity to normal human thyroid cells.

TT-1-induces apoptosis of TT cells in vitro. To investigate whether apoptosis was involved in TT-1-induced anti-TT activity, an AV/PI assay was conducted. The cells double-labeled with AV and PI, which discriminated between unaffected and apoptotic cells. AV-positive cells indicated the loss of membrane polarity, which leads to the complete loss of membrane integrity and subsequently, to apoptosis and PI infiltration. As shown in Fig. 2, treatment with TT-1 induced apoptosis of TT cells in a dose-dependent manner. Specifically, the average apoptotic cell accumulations reached 12.31, 18.62 and $31.07 \%$ for 2,4 and $8 \mu \mathrm{g} / \mathrm{ml}$ TT- 1 concentrations, respectively (Fig. 2B). Apoptosis may be one of the mechanisms by which TT-1 is able to prevent proliferation.

TT-1 upregulates Bax and downregulates Bcl-2 expression in TT cells. The Bcl-2 family of proteins is known to have critical roles in regulating apoptosis (28). Therefore, the authors of the present study examined the expression of Bax, the pro-apoptotic protein, and $\mathrm{Bcl}-2$, the anti-apoptotic protein, on the treatment of TT cells with TT-1 at the level of transcription and translation. As shown in Fig. 3A, the levels of Bax mRNA in TT cells was upregulated by TT- 1 treatment in a dose-dependent manner.

Additionally, treatment with TT-1 decreased the levels of Bcl-2 mRNA in TT cells in a dose-dependent manner (Fig. 3B). Specifically, at $8 \mu \mathrm{g} / \mathrm{ml}$ TT-1, the mRNA expression of $\mathrm{Bax}$ and $\mathrm{Bcl}-2$ were 2.23 -fold higher and 0.56 -fold lower, respectively compared with the control group. These changes were confirmed at the protein level using a western blot analysis (Fig. 3C-E).

TT-1 treatment activates the caspase-3 and -9 in TT cells. As apoptosis was observed in TT-1-treated TT cells, the present study measured the activity of different caspases as key factors of apoptosis using a fluorometric assay (Fig. 4). The results showed that caspase- 3 and -9 were activated in the TT-1-treated
TT cells in dose-dependent manners from $2-8 \mu \mathrm{g} / \mathrm{ml}$ (Fig. 4A and C). Furthermore, similar results were obtained in the RT-qPCR assays. The levels of caspase- 3 and -9 RNA in the TT cells increased following exposure to TT-1 (Fig. 4B and D).

TT-1 treatment suppresses the tumor growth in TT-bearing mice. To investigate whether TT-1 is able to inhibit tumor growth in vivo, a TT xenograft model was established. Following TT-1 treatment, the volume and weight of the tumors were measured. As indicated in Fig. 5A-C, TT-1 was able to suppress the tumor growth in nude mice in a dose dependent manner. Compared with the model group, the tumor inhibitory rates of the TT-1-treated groups were $30.00,34.28$ and $55.71 \%$ at treatment concentrations $0.04,0.2$ and $1 \mathrm{mg} / \mathrm{kg}$, respectively. The weight of the animals in the TT-1-treated groups to those of the model control group (Fig. 5D), indicating that there were no significant changes in body weight during TT-1 treatment. Therefore, cancer cell growth was significantly suppressed in mice treated with TT-1 without a significant loss in body weight.

\section{Discussion}

Thyroid cancer is the most frequent neoplasm of the endocrine system (2). The prognosis of thyroid cancer is excellent at the initial stages of disease. However, for advanced or metastatic diseases, limited therapeutic options are available (29). Additionally, conventional chemotherapy and radiotherapy often have severe side effects on healthy cells and tissues (30). As a result, the most promising drugs are those with low cytotoxicity, target selectivity and availability for chronic treatment.

Antimicrobial peptides have recently attracted significant attention as novel anticancer agents due to their novel mechanisms, decreased likelihood of drug resistance, and low intrinsic cytotoxicity (31). Melittin, which consists of 26 amino acid residues, is a cationic, hemolytic peptide isolated from honeybee venom. Previous studies have demonstrated that melittin has antibacterial, anti-arthritic and anti-inflammatory activities in various cell lines (6). Additionally, melittin has been shown to be a promising anticancer drug. A number of types of cancer cells, including renal, lung, breast, and bladder cells, have been reported to be selectively killed by melittin in vitro (14). In the present study, the authors designed a novel peptide, TT-1, based on the amphipathic structure of melittin. The peptide sequence of TT-1 was KIKAVLKVLTT, consisting of only 11 amino acids, which is much shorter than the peptide sequence length of melittin. The antimicrobial peptide database indicated that total hydrophobic ratio of TT-1 was 54\% and the net charge was 5 . These parameters indicated that TT-1 would be effective in treating cancer cells. Therefore, the relative activity and mechanism of TT-1 were further investigated.

It has been reported that melittin exhibits cytotoxic activity toward tumors and normal cells (32). In the present study, an in vitro study of the cytotoxic effect of TT-1 revealed that TT-1 was able to inhibit the proliferation of TT cells in a dose and time-dependent manner but had no significant growth inhibitory effects on normal thyroid follicular epithelial Nthy-ori3-1 cells. Therefore, TT-1 displayed selective anticancer activity. 



Figure 2. Induction of apoptosis by TT-1 in TT cells. (A) Cell apoptosis analysis of TT cells was conducted by flow cytometry using Annexin V/PI staining. Following the exposure of TT cells to $0-8 \mu \mathrm{g} / \mathrm{ml} \mathrm{TT}-1$ for $48 \mathrm{~h}$, the attached and detached cells were collected. Following staining with Annexin V and PI, the cells were subjected to flow cytometric analysis. Annexin $\mathrm{V}^{+} \mathrm{PI}^{+}$cells were considered to be late apoptotic cells, while Annexin $\mathrm{V}^{+} \mathrm{PI}{ }^{-}$cells were considered to be early apoptotic cells. (B) Bar chart showing the percentage of apoptosis. Respective data from $\geq 3$ independent experiments are summarized and presented as the mean \pm standard deviation. ${ }^{* *} \mathrm{P}<0.01$ vs. the control group. PI, propidium iodide.

A

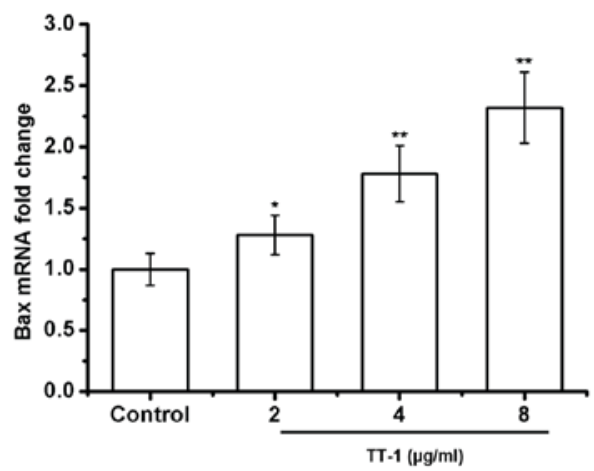

B

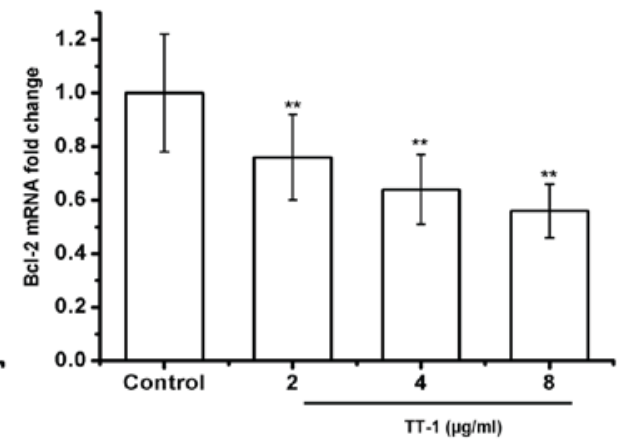

$E$

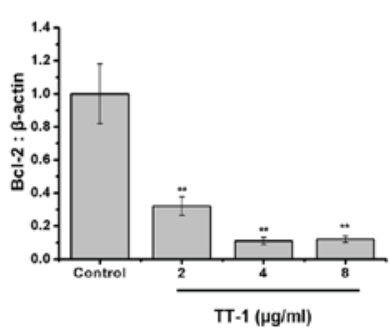

Figure 3. TT-1 induces changes in expression of mitochondrial-dependent proteins. (A and B) Quantitative reverse transcription polymerase chain reaction of apoptosis-associated genes in TT cells demonstrated significant (A) upregulation of Bax and (B) downregulation of Bcl-2 at the mRNA level. The data represent the mean \pm standard deviation of three independent experiments. ${ }^{*} \mathrm{P}<0.05,{ }^{* *} \mathrm{P}<0.01$ vs. the untreated group. (C) Western blot analysis confirmed the upregulation of Bax and downregulation of Bcl-2 at the protein level. Densitometric analysis of Bax (D) or Bcl-2 (E) protein levels. ${ }^{* *} \mathrm{P}<0.01$ vs. the control group. Western blots are representative blots from three independent experiments with similar results. 
A

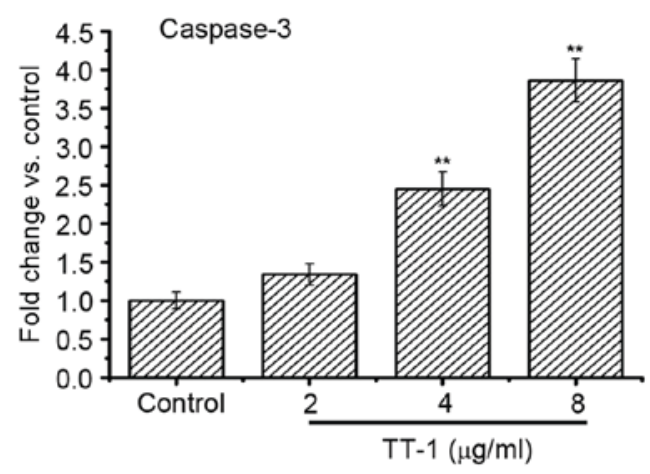

C

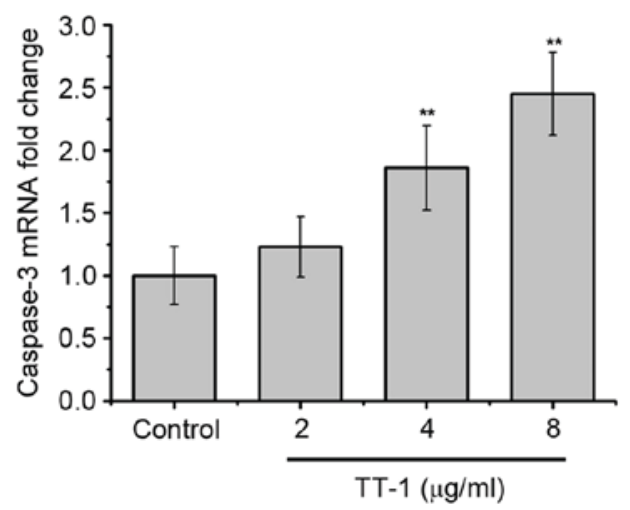

B

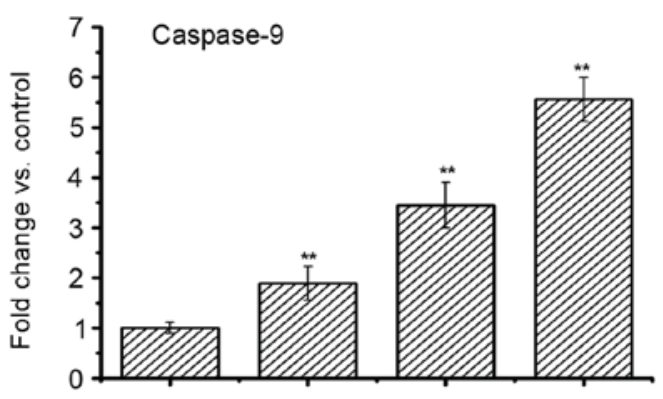

D

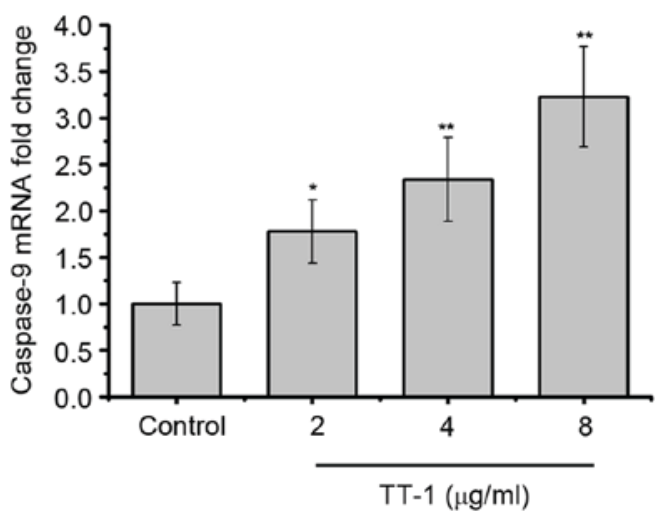

Figure 4. TT-1 activates caspase- 3 and -9 in TT cells. The cells were treated with $0-8 \mu \mathrm{g} / \mathrm{ml}$ TT- 1 for $48 \mathrm{~h}$. The activity of caspase- 3 and -9 was measured. TT- 1 increases the levels of (A) active caspase-3 and (B) active caspase-9. TT-1 upregulates (C) caspase- 3 and (D) caspase-9 gene expression. Data are presented as the mean \pm standard deviation; $\mathrm{n}=3 ;{ }^{*} \mathrm{P}<0.05,{ }^{* * *} \mathrm{P}<0.01$ vs. the untreated group.

A

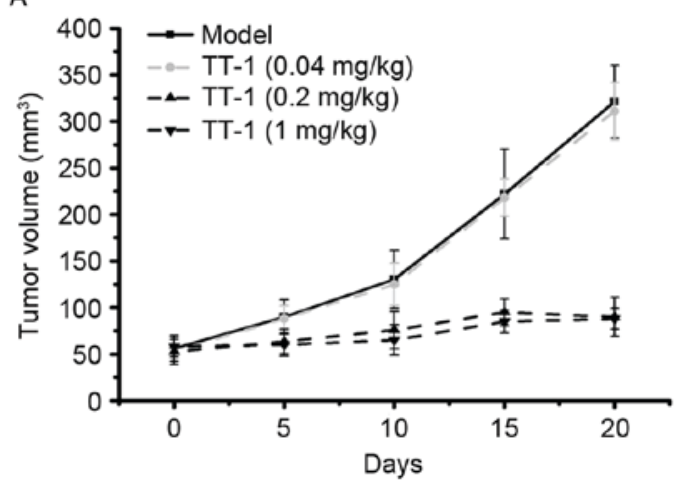

C

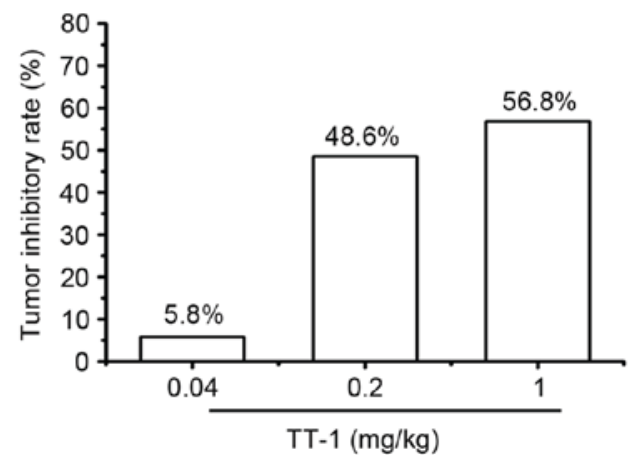

B



D



Figure 5. TT-1 prevents TT tumor growth in nude mice. (A) A tumor volume curve in TT-bearing mice treated with or without TT-1. (B) Weight of tumors in each group. (C) Tumor inhibitory rates of each experimental group. (D) The body weight curves of each group. Data are presented as the mean \pm standard deviation; $\mathrm{n}=10 ;{ }^{* *} \mathrm{P}<0.01$ vs. the control group. 
Apoptosis, a very tightly programmed cell death with distinct biochemical and genetic pathways (33), is generally identified via specific morphological cellular characteristics, including cell shrinkage, nuclear or cytoplasmic fragmentation and chromatin condensation (34). A class of cysteine proteases, including caspase-3, -8 and -9 , is commonly involved in the apoptotic pathways (35). On the other hand, the Bcl-2 family also has an important role in the regulation of apoptosis (36). Defects in apoptotic mechanisms have important roles in tumor pathogenesis, which allows neoplastic cells to survive over intended lifespans, subverts the need for exogenous survival factors and provides protection from oxidative stress and hypoxia as the tumor mass expands (32). Therefore, the ability to induce apoptosis is necessary for effective anticancer therapies (37). In the present study, double staining of cells with AV/PI revealed that TT-1-induced apoptosis may be one of the mechanisms by which TT-1 prevents the growth of TT cells in vitro.

Bcl-2 and Bax, members of the Bcl-2 family of proteins, are important components of ischemia-reperfusion injury-induced apoptosis (28). These two proteins can form either homodimers or heterodimers, which depends on the levels of each component that is present. Bax forms a heterodimer with Bcl-2 and functions as an apoptotic activator by increasing the opening of the mitochondrial voltage-dependent anion channel, which leads to the loss in membrane potential (38). Therefore, high expression of $\mathrm{Bcl}-2$ is able to inhibit apoptosis, while high expressions of Bax can stimulate apoptosis. A change in the expression ratio of these two factors determines whether apoptosis occurs (39). In the present study, the results shown in Fig. 3 suggest that the apoptotic mechanism of TT-1 in TT cells include the downregulation of $\mathrm{Bcl}-2$ expression and the upregulation of Bax expression at the level of transcription and translation.

Additionally, caspase family members have major roles in cell apoptosis (40). The caspase cleavage cascade begins with initiator caspase being activated by intrinsic or extrinsic pathways. In the present study, the authors examined two typical caspase family members, caspase-3 and -9. Caspase- 3 is an effector caspase that mediates the cleavage of many proteins, while caspase- 9 is the key initiator caspase in the intrinsic pathway that induces cell death and the activation of which occurs at the mitochondrial membrane (41). The present study showed that caspase-3 and -9 mRNA levels were significantly increased in the TT-1-treated group compared with the untreated controls and indicated that TT-1 may induce the apoptosis of TT cells, which may be partly due to the activation of caspase-3 and -9 (Fig. 4).

From these results, TT-1 exhibited a marked inhibitory effect on cell viability on TT cells in vitro, and it was also demonstrated that TT-1 exhibited anti-tumor activity on TT cells in vivo (Fig. 5). Compared with the control, TT-1 was able to suppress the proliferation of TT cells tumor-bearing mice in a dose dependent manner, with an observed $55.71 \%$ inhibition at $1 \mathrm{mg} / \mathrm{kg}$ TT-1. This finding was further confirmed by results of TT tumor growth in vivo, and TT-1 treatment had no effect on the weight of mice, indicating that TT-1 may be a potential high efficiency and low toxicity anticancer agent.

In summary, TT-1 inhibited the proliferation of human TT cells in vitro and in vivo through the upregulation of Bax, the downregulation of Bcl-2 and the activation of caspase-3 and -9 . These results further suggested that TT-1 may be a potential candidate for the treatment of thyroid cancer.

\section{Acknowledgements}

This study was financially supported by a grant fromJilin Provincial Finance Department (grant no. SCZSY201504) and the Outstanding Young Talent Foundation Project of Science and Technology Department in Jilin Province (grant no. 20170520018JH), China.

\section{References}

1. Yan JX, Wang KR, Chen R, Song JJ, Zhang BZ, Dang W, Zhang W and Wang R: Membrane active antitumor activity of NK-18, a mammalian NK-lysin-derived cationic antimicrobial peptide. Biochimie 94: 184-191, 2012.

2. Pellegriti G, Frasca F, Regalbuto C, Squatrito S and Vigneri R: Worldwide increasing incidence of thyroid cancer: Update on epidemiology and risk factors. J Cancer Epidemiol 2013: 965212, 2013.

3. Jemal A, Bray F, Center MM, Ferlay J, Ward E and Forman D: Global cancer statistics. CA Cancer J Clin 6: 69-90, 2011.

4. Matsuno A, Murakami M, Hoya K, Yamada SM, Miyamoto S, Yamada S, Son JH, Nishido H, Ide F, Nagashima $H$, Sugaya M, et al: Clinicopathological and molecular histochemical review of skull base metastasis from differentiated thyroid carcinoma. Acta Histochem Cytochem 46: 129-136, 2013.

5. Xing M, Haugen BR and Schlumberger M: Progress in molecular-based management of differentiated thyroid cancer. Lancet 381: 1058-1069, 2013.

6. Brown CK and Kirkwood JM: Medical management of melanoma. Surg Clin North Am 83: 283-322, viii, 2003.

7. Lienkamp K and Tew GN: Synthetic mimics of antimicrobial peptides-aversatile ring-opening metathesis polymerization based platform for the synthesis of selective antibacterial and cell-penetrating polymers. Chemistry 15: 11784-11800, 2009.

8. Chen C, Hu J, Zhang S, Zhou P, Zhao X, Xu H, Zhao X, Yaseen M and Lu JR: Molecular mechanisms of antibacterial and antitumor actions of designed surfactant-like peptides. Biomatials 33: 592-603, 2012.

9. Brogden KA: Antimicrobial peptides: Pore formers or metabolic inhibitors in bacteria? Nav Rev Microbiol 3: 238-250, 2005.

10. Mader JS and Hoskin DW: Cationic antimicrobial peptides as novel cytotoxic agents for cancer treatment. Expert Opin Investig Grugs 15: 933-946, 2006.

11. Takahashi T, Nomura F, Yokoyama Y, Tanaka-Takiguchi Y, Homma $\mathrm{M}$ and Takiguchi $\mathrm{K}$ : Multiple membrane interactions and versatile vesicle deformations elicited by melittin. Toxins 5 : 637-664, 2013.

12. Han SM, Kim JM, Park KK, Chang YC and Pak SC: Neuroprotective effects of melittin on hydrogen peroxide-induced apoptotic cell death in neuroblastoma SH-SY5Y cells. BMC Complement Altern Med 14: 286, 2014.

13. Sommer A, Fries A, Cornelsen I, Speck N, Koch-Nolte F, Gimpl G, Andrä J, Bhakdi S and Reiss K: Melittin modulates keratinocyte function through P2 Receptor-dependent ADAM Activation. J Biol Chem 287: 23678-23689, 2012.

14. Son DJ, Lee JW, Lee YH, Song HS, Lee CK and Hong JT: Therapeutic application of anti-arthritis, pain-releasing and anticancer effects of bee venom and its constituent compounds. Pharmacol Ther 115: 246-270, 2007.

15. Oršolić N: Bee venom in cancer therapy. Cancer Metastasis Rev 31: 173-194, 2012.

16. Raghuraman $\mathrm{H}$ and Chattopadhyay A: Melittin: A membrane-active peptide with diverse functions. Biosci Rep 27: 189-223, 2007.

17. Li SA, Lee WH and Zhang Y: Efficacy of OH-CATH30 and its analogs against drug-resistant bacteria in vitro and in mouse models. Antimicrob Agents Chemother 56: 3309-33017, 2012.

18. MaQ,Jiao W,Lv Y,Dong N,Zhu X and Shan A: Structure-function relationship of Val/Arg-rich peptides: Effects of net charge and pro on activity. Chem Biol Drug Des 84: 348-353, 2014.

19. Ozgur B and Sayar M: Role of hydrophobic/aromatic residues on the stability of double-wall $\beta$-sheet structures formed by a triblock peptide. J Phys Chem B 121: 4115-4128, 2017. 
20. Starenki D and Park JI: Mitochondria-targeted nitroxide, mito-CP, suppresses medullary thyroid carcinoma cell survival in vitro and in vivo. J Clin Endocrinol Metab 98: 1529-15240, 2013.

21. Wang H, Ke M, Tian Y, Wang J, Li B, Wang Y, Dou J and Zhou C: BF-30 selectively inhibits melanoma cell proliferation via cytoplasmic membrane permeabilization and DNA-binding in vitro and in B16F10-bearing mice. Eur J Pharmaco 707: 1-10, 2013.

22. Paredes-Gamero EJ, Martins MN, Cappabianco FA, Ide JS and Miranda A: Characterization of dual effects induced by antimicrobial peptides: Regulated cell death or membrane disruption. Biochim Biophys Acta 1820: 1062-91072, 2012.

23. Massaoka MH, Matsuo AL, Figueiredo CR, Farias CF, Girola N, Arruda DC, Scutti JA, Romoff P, Favero OA, Ferreira MJ, et al: Jacaranone induces apoptosis in melanoma cells via ROS-mediated down regulation of Akt and p38 MAPK activation and displays antitumor activity in vivo. PLoS One 7: e38698, 2012.

24. Wu C, Geng X, Wan S, Hou H, Yu F, Jia B and Wang L: Cecropin-P17, an analog of Cecropin B, inhibits human hepatocellular carcinoma cell HepG-2 proliferation via regulation of ROS, Caspase, Bax, and Bcl-2. J Pept Sci 21: 661-668, 2015.

25. Lu J, Yao YY, Dai QM, Ma GS, Zhang SF, Cao L, Ren LQ and Liu NF: Erythropoietin attenuates cardiac dysfunction by increasing myocardial angiogenesis and inhibiting interstitialfibrosis in diabetic rats. Cardiovasc Diabetol 11: 105, 2012.

26. Tano T, Okamoto M, Kan S, Nakashiro K, Shimodaira S, Koido S, Homma S, Sato M, Fujita T, Kawakami Y and Hamakawa $\mathrm{H}$ : Prognostic Impact of Expression of Bcl-2 and Bax Genes in Circulating Immune Cells Derived from Patients with Head and Neck Carcinoma. Neoplasia 15: 305-314, 2013

27. Livak KJ and Schmittgen TD: Analysis of relative gene expression data using real-time quantitative PCR and the 2(-Delta Delta C(T)) method. Methods 25: 402-408, 2001.

28. Ng CS, Wan S and Yim AP: Pulmonary ischemia-reperfusion in-jury: Role of apoptosis. Eur Respir J 25: 356-363, 2005.

29. Stjepanovic N and Capdevila J: Multikinase inhibitors in the treatment of thyroid cancer: Specific role of lenvatinib. Biologics 8: 129-139, 2014

30. Leung HW, Yang WH, Lai MY, Lin CJ and Lee HZ: Inhibition of 12-lipoxygenase during baicalein-induced human lung non-small carcinoma H460 cell apoptosis. Food Chem Toxicol 45: 403-411, 2007.
31. Schweizer F: Cationic amphiphilic peptides with cancer selective toxicity. Eur J Pharmacol 625: 190-194, 2007.

32. Sun D, Sun M, Zhu W, Wang Z, Li Y and Ma J: The anti-cancer potency and mechanism of a novel tumor-activated fused toxin, DLM. Toxins (Basel) 7: 423-438, 2015.

33. Lockshin RA and Williams CM: Programmed cell death-I. Cytology of degeneration in the intersegmental muscles of the Pernyi silkmoth. J Insect Physiol 11: 123-133, 1965.

34. Bottone MG, Santin G, Aredia F, Bernocchi G, Pellicciari C and Scovassi AI: Morphological features of organelles during apoptosis: An overview. Cells 2: 294-305, 2013.

35. Nuñez G, Benedict MA, Hu Y and Inohara N: Caspases: The proteases of the apoptotic pathway. Oncogene 17: 3237-3245, 1998.

36. Ji ES, Kim YM, Shin MS, Kim CJ, Lee KS, Kim K, Ha J and Chung YR: Treadmill exercise enhances spatial learning ability through suppressing hippocampal apoptosis in Huntington's disease rats. J Exerc Rehabil 11: 133-139, 2015.

37. Tseng TH, Shen CH, Huang WS, Chen CN, Liang WH, Lin TH and Kuo HC: Activation of neutral-sphingomyelinase, MAPKs, and p75 NTR-mediating caffeic acid phenethyl ester-induced apoptosis in C6 glioma cells. J Biomed Sci 21: 61, 2014.

38. Vadde R, Radhakrishnan S, Reddivari L and Vanamala JK: Triphala extract suppresses proliferation and induces apoptosis in human colon cancer stem cells via suppressing c-Myc/Cyclin D1 and Elevation of Bax/Bcl-2 Ratio. Biomed Res Int 2015: 649263, 2015.

39. Zhang C, Guo Z, Liu H, Shi Y and Ge S: Influence of levosimendan postconditioning on apoptosis of rat lung cells in a model of ischemia-reperfusion injury. PLoS One 10: e0114963, 2015.

40. Floyd DH, Zhang Y, Dey BK, Kefas B, Breit H, Marks K, Dutta A, Herold-Mende C, Synowitz M, Glass R, et al: Novel anti-apoptotic microRNAs 582-5p and 363 promote human glioblastoma stem cell survival via direct inhibition of caspase 3 , caspase 9 and Bim. PLoS One 9: e96239, 2014.

41. Wyllie AH: 'Where, $\mathrm{O}$ death, is thy sting?' A brief review of apoptosis biology. Mol Neurobiol 42: 4-9, 2010. 